Journal of Mathematics and Informatics

Vol. 10, 2017, 41-48

ISSN: 2349-0632 (P), 2349-0640 (online)

Published 11 December 2017

www.researchmathsci.org

DOI: http://dx.doi.org/10.22457/jmi.v10a6

Journal of

Mathematics and

Informatics

\title{
On the Non-Homogeneous Quadratic Equation with Five Unknowns $x^{2}+x y-y^{2}-(z+w)=10 p^{2}$
}

K.Dhivya ${ }^{1}$ and T.R.Usha Rani ${ }^{2}$

${ }^{1}$ Department of Mathematics, Shrimati Indira Gandhi College, Trichy-620002

Tamilnadu, India; e-mail:kdhivya10495@gmail.com

${ }^{2}$ Department of Mathematics, Shrimati Indira Gandhi College, Trichy-620002

Tamilnadu, India; e-mail:usharanisigc@gmail.com

Received 2 November 2017; accepted 5 December 2017

Abstract. The non-homogeneous quadratic equation with five unknowns represented by the Diophantine equation $x^{2}+x y-y^{2}-(z+w)=10 p^{2}$ is analysed for its non-zero distinct integral solutions. Various interesting relations between the solutions and special numbers are exhibited.

Keywords: non-homogeneous quadratic, quadratic with five unknowns, integral solutions.

\section{AMS Mathematics Subject Classification (2010): 11D09}

\section{Introduction}

The non-homogenous quadratic Diophantine equation offers an unlimited field for research because of their variety [1-3]. For an extensive review of various problems one may refer [4-17]. This communication concerns with yet another interesting equation $x^{2}+x y-y^{2}-(z+w)=10 p^{2}$ representing non-homogeneous quadratic equation with five unknowns determining its infinitely many non-zero integral solutions. Also a few interesting properties among the solutions are presented.

\section{Notation}

$$
\begin{array}{ll}
t_{m, n}=n\left(1+\frac{(n-1)(m-2)}{2}\right) & \text { - Polygonal number of rank } n \text { with sides } m \\
C t_{m, n}=\frac{m n(n-1)+2}{2} & \text { - Centered Polygonal number of rank } n \text { with sides } m \\
S_{n}=6 n(n-1)+1 & \text { - Star number of rank } n \\
P R_{n}=n(n+1) & \text { - Pronic number of rank } n
\end{array}
$$




\section{K.Dhivya and T.R.Usha Rani}

$j_{n}=2^{n}+(-1)^{n}$

$G_{n}=2 n-1$

$S o_{n}=n\left(2 n^{2}-1\right)$
- Jacobsthal-Lucas number of rank $n$

- Gnomonic number of rank $n$

- Stella Octangular number of rank $n$

\section{Method of analysis}

The non-homogeneous quadratic equation with five unknowns under consideration is,

$$
x^{2}+x y-y^{2}-(z+w)=10 p^{2}
$$

We have four patterns of solutions of (1) which are presented below.

The substitution of linear transformation

$$
x=u+v, y=u-v, z=2 u v+1, w=2 u v-1
$$

in (1) leads to

$$
u^{2}=10 p^{2}+v^{2}
$$

\subsection{PATTERN I}

Assume that $p=2 m n, u=10 m^{2}+n^{2}, v=10 m^{2}-n^{2}$

Substituting the values of $u$ and $v$ in (1) and simplifying, we obtain the non-zero distinct integer solutions of (1) as follows.

$$
\begin{aligned}
& x(m)=20 m^{2} \\
& y(n)=2 n^{2} \\
& z(m, n)=200 m^{4}-2 n^{4}+1 \\
& w(m, n)=200 m^{4}-2 n^{4}-1 \\
& p(m, n)=2 m n
\end{aligned}
$$

\section{PROPERTIES}

1. Each of the following expressions is a Nasty number:
(i) $3[z(n, n)-w(n, n)]$
(ii) $3 y(n)$
(iii) $30[x(m)]$
(iv) $30[z(m, n)]-30 x\left(3 m^{2}\right)+30 y\left(n^{2}\right)-30$
$2.20[z(m, n)+w(m, n)]=x\left(20 m^{2}\right)-10 y\left(2 n^{2}\right)$
3. $p(n, n(n+1))-S O_{n}-n G_{n} \equiv 0(\bmod 2)$
4. $p(n, n)+y(n)-4 P R_{n}-G_{n}-S_{n}+6 t_{4, n}=0$
5. $x(n)-p(n, n)-2 P R_{n}+G_{n}-16 t_{4, n}+1=0$ 
On the Non-Homogeneous Quadratic Equation with Five Unknowns

$$
x^{2}+x y-y^{2}-(z+w)=10 p^{2}
$$

6. $p(n, n)+y(n)-4 t_{4, n}=0$

7. $x\left(2^{n}\right)+y\left(2^{n}\right)-22 j_{2 n}+22=0$

8. $p\left(2^{n}, 2^{n}\right)-2 j_{2 n}+2=0$

9. $w\left(2^{n}, 1\right)-200 j_{4 n}+203=0$

10. Each of the following expressions is a biquadratic integer:
(1) $\frac{8[z(m, 1)+1]}{100}$,
(2) $\frac{8[w(m, 1)+3]}{100}$

\subsection{PATTERN II}

(3) can be written as,

$$
u^{2}-v^{2}=10 p^{2}
$$

(4) can be written in the form of ratio as

$$
\begin{aligned}
& u^{2}-v^{2}=10 p \cdot p \\
& \frac{u+v}{p}=\frac{10 p}{u-v}=\frac{\alpha}{\beta}, \beta \neq 0
\end{aligned}
$$

which is equivalent to the system of double equations

$$
\begin{aligned}
& p \alpha-u \beta-v \beta=0 \\
& 10 p \beta-u \alpha+v \alpha=0
\end{aligned}
$$

Solving (7) and (8) by the method of cross multiplication, we obtain,

$$
p=-2 \alpha \beta, u=-\left(\alpha^{2}+10 \beta^{2}\right), v=10 \beta^{2}-\alpha^{2}
$$

Substituting the values of $u$ and $v$ in (2) and simplifying, we obtain the non-zero distinct integer solutions of (1) as follows.

$$
\begin{aligned}
& x(\alpha)=-2 \alpha^{2} \\
& y(\beta)=-20 \beta^{2} \\
& z(\alpha, \beta)=2 \alpha^{4}-200 \beta^{4}+1 \\
& w(\alpha, \beta)=2 \alpha^{4}-200 \beta^{4}-1 \\
& p(\alpha, \beta)=-2 \alpha \beta
\end{aligned}
$$

\section{PROPERTIES}

1. Each of the following expressions is a Nasty number:

(i) $3[z(\alpha, \beta)-w(\alpha, \beta)]$

(ii) $3[x(\alpha)-y(\alpha)]$

2. $20[z(\alpha, \beta)+w(\alpha, \beta)]=y\left(20 \beta^{2}\right)-10 x\left(2 \alpha^{2}\right)$ 


\section{K.Dhivya and T.R.Usha Rani}

3. $p(n, n(n+1))+S O_{n}+n G_{n}+G_{n}-1 \equiv 0(\bmod 2)$

4. $p(n, n)-y(n)-2 P R_{n}+G_{n}-16 t_{4, n}+1=0$

5. $x(\alpha)+p(\alpha, \alpha)+4 P R_{\alpha}-S_{\alpha}+6 t_{4, \alpha}+1 \equiv 0(\bmod 10)$

6. $x(\alpha)+p(\alpha, \alpha)+4 t_{4, \alpha}=0$

7. $z\left(2^{n}, 2^{n}\right)+w\left(2^{n}, 2^{n}\right)+396 j_{4 n}-396=0$

8. $z\left(2^{n}, 1\right)-2 j_{4 n}+201=0$

9. $x\left(2^{n}\right)-y\left(2^{n}\right)-18 j_{2 n}+18=0$

10. Each of the following expressions is a biquadratic integer:
(1) $\frac{8[z(1, \beta)-3]}{100}$
(2) $\frac{8[w(1, \beta)-1]}{100}$

NOTE:

(4) can also be written as system of double equations

$$
\begin{gathered}
u+v=10 p \\
u-v=p
\end{gathered}
$$

Solving (9) and (10) we obtain,

$$
\left.\begin{array}{c}
u=\frac{11 p}{2} \\
v=\frac{9 p}{2}
\end{array}\right\}
$$

To obtain the integer solutions, take $p=2 k$ in (11) we get,

$$
\left.\begin{array}{c}
u=11 k \\
v=9 k
\end{array}\right\}
$$

Substituting (12) in (2) and simplifying, we obtain the non-zero integer solutions of (1) are given by,

$$
\begin{aligned}
& x(k)=20 k \\
& y(k)=2 k \\
& z(k)=198 k+1 \\
& w(k)=198 k-1 \\
& p(k)=2 k
\end{aligned}
$$

\subsection{PATTERN III}

(4) can be written as,

$$
(u+v)(u-v)=2 p .5 p
$$


On the Non-Homogeneous Quadratic Equation with Five Unknowns

$$
x^{2}+x y-y^{2}-(z+w)=10 p^{2}
$$

(13) can be written in the form of ratio as,

$$
\frac{u+v}{2 p}=\frac{5 p}{u-v}=\frac{\alpha}{\beta}, \beta \neq 0
$$

which is equivalent to the system of double equations.

$$
\begin{aligned}
& 2 p \alpha-u \beta-v \beta=0 \\
& 5 p \beta-u \alpha+v \beta=0
\end{aligned}
$$

Solving (15) and (16) by the method of cross multiplication, we obtain,

$$
p=-2 \alpha \beta, u=-\left(5 \alpha^{2}+2 \beta^{2}\right), v=-5 \alpha^{2}+2 \beta^{2}
$$

Substituting the values of $u$ and $v$ in (2) and simplifying, we obtain the non-zero distinct integer solutions of (1) as follows.

$$
\begin{aligned}
& x(\alpha)=-10 \alpha^{2} \\
& y(\beta)=-4 \beta^{2} \\
& z(\alpha, \beta)=50 \alpha^{4}-8 \beta^{4}+1 \\
& w(\alpha, \beta)=50 \alpha^{4}-8 \beta^{4}-1 \\
& p(\alpha, \beta)=-2 \alpha \beta
\end{aligned}
$$

\section{PROPERTIES}

1. Each of the following expressions is a Nasty number:
(i) $y(n)-x(n)$
(ii) $3[p(n, n)-y(n)]$

2. $20[z(\alpha, \beta)+w(\alpha, \beta)]=5 y\left(4 \beta^{2}\right)-2 x\left(10 \alpha^{2}\right)$

3. $y(n)+p(n, n)-x(n)-4 P R_{n}-G_{n}-S_{n}+6 t_{4, n}=0$

4. $x(n)-p(n, n)+2 C t_{8, n}+16 P R_{n}-2 t_{18, n}-2 \equiv 0(\bmod 22)$

5. $p(n, n)-y(n)-2 P R_{n}+G_{n}+1=0$

6. $p(n, n)+y(n)-x(n)-4 t_{4, n}=0$

7. $z\left(2^{n}, 1\right)-50 j_{4 n}+57=0$

8. $z\left(2^{n}, 1\right)-w\left(2^{n}, 1\right)-100 j_{4 n}+98=0$

9. $y\left(2^{n}\right)-x\left(2^{n}\right)-6 j_{4 n}+6=0$

10. Each of the following expressions is a biquadratic integer:
(i) $2[z(1, \beta)-51]$
(ii) $2[w(1, \beta)-49]$

\section{NOTE:}

(13) can also be written as system of double equations

$$
\begin{aligned}
& u+v=5 p \\
& u-v=2 p
\end{aligned}
$$




\section{K.Dhivya and T.R.Usha Rani}

Solving (17) and (18) we obtain

$$
\left.\begin{array}{l}
u=\frac{7 p}{2} \\
v=\frac{3 p}{2}
\end{array}\right\}
$$

To obtain the integer solutions, take $p=2 k$ in (19) we get,

$$
\left.\begin{array}{l}
u=7 k \\
v=3 k
\end{array}\right\}
$$

Substituting (20) in (2) and simplifying, we obtain the non-zero distinct integer solution are given by,

$$
\begin{aligned}
& x(k)=10 k \\
& y(k)=4 k \\
& z(k)=42 k^{2}+1 \\
& w(k)=42 k^{2}-1 \\
& p(k)=2 k
\end{aligned}
$$

\subsection{PATTERN IV}

(4) can be written as,

$$
(u+v)(u-v)=2.5 p^{2}
$$

(21) can be written as system of double equations

$$
\begin{aligned}
& u+v=5 p^{2} \\
& u-v=2
\end{aligned}
$$

Solving (22) and (23) we obtain

$$
\left.\begin{array}{l}
u=\frac{5 p^{2}+2}{2} \\
v=\frac{5 p^{2}-2}{2}
\end{array}\right\}
$$

To obtain the integer solutions, take $p=2 k$ in (24) we get,

$$
\left.\begin{array}{l}
u=10 k^{2}+1 \\
v=10 k^{2}-1
\end{array}\right\}
$$

Substituting (25) in (2) and simplifying, we obtain the non-zero distinct integer solution of (1) as follows.

$$
\begin{aligned}
& x(k)=20 k^{2}, \quad y=2, \quad z(k)=200 k^{4}-3 \\
& w(k)=200 k^{4}-1, \quad p(k)=2 k
\end{aligned}
$$

\section{PROPERTIES}

1. Each of the following expressions is a Nasty number: 
On the Non-Homogeneous Quadratic Equation with Five Unknowns

$$
x^{2}+x y-y^{2}-(z+w)=10 p^{2}
$$

$$
\begin{aligned}
& \text { (i) } 30[x(k)], \quad \text { (ii) } 6 y\left(k^{2}\right)+6 p\left(k^{2}\right) \\
& \text { 2. } 20[z(k)+w(k)]=x\left(20 k^{2}\right)+20 y \\
& \text { 3. } x(k)+y\left(k^{2}\right)-p\left(k^{2}\right)-2 P R_{k}+G_{k}-18 t_{4, k}+1=0 \\
& \text { 4. } x(k)-p\left(k^{2}\right)-2 P R_{k}+G_{k}-16 t_{4, k}+1=0 \\
& \text { 5. } y\left(k^{2}\right)+p\left(k^{2}\right)-4 P R_{k}-G_{k}-S_{k}+6 t_{4, k}=0 \\
& \text { 6. } x(k)+y\left(k^{2}\right)+p\left(k^{2}\right)-24 t_{4, k}=0 \\
& \text { 7. } z\left(2^{n}\right)+w\left(2^{n}\right)-400 j_{4 n}+404=0 \\
& \text { 8. } x\left(2^{n}\right)+p\left(2^{n}\right)-20 j_{2 n}+20-2 j_{n}+2(-1)^{n}=0 \\
& \text { 9. } x\left(2^{n}\right)+z\left(2^{n}\right)+w\left(2^{n}\right)-20 j_{2 n}-400 j_{4 n}+423=0
\end{aligned}
$$

10. Each of the following expression is a biquadratic integer:

$$
\text { (i) } \frac{8[z(k)+3]}{100} \text {, (ii) } \frac{8[w(k)+3]}{100}
$$

\section{Conclusion}

In this paper we have made an attempt to obtain all integer solutions to the nonhomogeneous quadratic equation. One may search for other patterns of solutions and their corresponding properties.

\section{REFERENCES}

1. L.E.Dickson, History of Theory of Numbers and Diophantine Analysis, Dove Publications, New York, Vol 2 (2005).

2. L.J.Mordell, Diophantine Equations, Academic press, New York, (1970).

3. R.D.Carmicheal, The Theory of Numbers and Diophantine analysis, Dover Publications, New York, (1959).

4. M.A.Gopalan and D.Geetha, Lattice points on the Hyperboloid of two sheets $x^{2}-6 x y+y^{2}+6 x-2 y+5=z^{2}+4$, Impact J. Sci. Tech., 4 (2010) 23-32.

5. M.A.Gopalan, S.Vidhyalakshmi and S.Kavitha, Integral points on the Homogeneous Cone $z^{2}=2 x-7 y^{2}$, Diophantus J. Math., 1(2) (2012) 127-136.

6. M.A.Gopalan, S.Vidhyalakshmi and G.Sumathi, Lattice points on the Hyperboloid of one sheet $4 z^{2}=2 x^{2}+3 y^{2}-4$, Diophantuts J. Math., 1(2) (2012) 109-115.

7. M.A.Gopalan, S.Vidhyalakshmi and K.Lakshmi, Integral Points on the Hyperboliodof two sheets $3 y^{2}=7 x^{2}-z^{2}+21$, Diophantus J Math., 1(2) (2012) 99-107. 
K.Dhivya and T.R.Usha Rani

8. M.A.Gopalan, S.Vidhyalakshmi and S.Mallika, Observations on Hyperboloid of one sheet $x^{2}+2 y^{2}-z^{2}=2$, Bessel J Math., 2(3) (2012) 221-226.

9. M.A.Gopalan, S.Vidhyalakshmi, T.R.Usha Rani and S.Mallika, Integral Points on the Homogeneous Cone $6 z^{2}+3 y^{2}-2 x^{2}=0$, The Impact J. Sci. Tech., 6(1) (2012) 7-13.

10. M.A.Gopalan,S.Vidhyalakshmi and T.R.UshaRani, Integral points on the nonhomogeneous Cone $2 z^{2}+4 x y+8 x-4 z=0$, Global Journal of Mathematics, Mathematical Sciences, 2(1) (2010) 61-67.

11. M.A.Gopalan, S.Vidhyalakshmi and J.Uma Rani, Intergal points on the Homogeneous Cone $4 y^{2}+x^{2}=37 z^{2}$, Cayley J. of Math., 2(2) (2013) 101-107.

12. M.A.Gopalan, S.Vidhyalakshmi and A.Kavitha, Observations on the Hyperboloid of two sheet $7 x^{2}-3 y^{2}=z^{2}+z(y-x)+4$, International Journal of the Latest Research in Scienceand Technology, 2(2) (2013) 84-86.

13. M.A.Gopalan and B.Sivagami, Integral points on the Homogeneous Cone $z^{2}=3 x^{2}+6 y^{2}$, IOSR Journal of Mathematics, 8(4) (2013) 24-29.

14. M.A.Gopalan and V.Geetha, Lattice points on the Homogeneous Cone $z^{2}=2 x^{2}+8 y^{2}-6 x y$, Indian Journal of Science, 2 (2013) 93-96.

15. M.A.Gopalan, S.Vidhyalakshmi and J.Uma Rani, On the ternary quadratic diophantine equation $6\left(x^{2}+y^{2}\right)-8 x y=21 z^{2}$.,Sch. Eng. Tech., 2(2A) (2014) 108112.

16. M.A.Gopalan, S.Vidhyalakshmi and J.Shanthi, Lattice Points on the Homogeneous Cone $8\left(x^{2}+y^{2}\right)-15 x y=56 z^{2}$, Sch. Journal of Phy. Math. Star., 1(1) (2014) 29-32.

17. K.Meena, M.A.Gopalan, E.Bhuvaneshwari and R.Presenna, On quadratic diophantine equation with five unknowns $4 w^{2}-x^{2}-y^{2}+z^{2}=t^{2}$, International Journal of Advanced Scientific Research, 1(2) (2016) 62-64. 